\title{
Evidence for an intrinsic control of myometrial contractile periodicity in sheep during pregnancy
}

\author{
S. J. Lye \\ Division of Perinatology, Samuel Lunenfeld Research Institute, Department of Obstetrics and \\ Gynaecology, Mount Sinai Hospital, and Departments of Obstetrics and Gynaecology and of \\ Physiology, University of Toronto, Canada
}

\begin{abstract}
Summary. Reduction in concentrations of prostaglandins in plasma by administration of sodium meclofenamate to pregnant sheep failed to alter the frequency or duration of electromyographic activity bursts or the response to oxytocin of myometrial tissue transplanted to the omentum. However, a significant $(P<0.05)$ delay $(8.6 \pm 3.8$ versus $1.3 \pm 0.3 \mathrm{~min}$ ) in the myometrial response to oxytocin was observed when the hormone was administered $1 \mathrm{~min}$ after a spontaneous burst of electromyographic activity compared with $15 \mathrm{~min}$ after a burst, indicating a period of refractoriness. Similarly, the myometrial threshold for electrical stimulation was higher at $10-25 \%$ of the interval between contractions than close to the expected time of the next contraction. Stimulation of the myometrium at intervals of $30 \mathrm{~s}$ revealed a cycling of the electrical stimulation threshold: significantly higher voltages were required to elicit responses between spontaneous bursts of electromyographic activity $(18 \cdot 0 \pm 2 \cdot 2 \mathrm{~V})$ than during bursts $(11.3 \pm 1 \cdot 6 \mathrm{~V})$. In contrast, there was no voltage differential in animals close to labour $(<24 \mathrm{~h})$. These data provide no evidence to support a role for prostaglandins in the generation of contractions during pregnancy, but suggest that periodicity of contractions is associated with inherent changes in myometrial responsiveness to stimulation, which could occur as a result of a cycling of the resting membrane potential.
\end{abstract}

Keywords: uterine contractions; myometrium; pregnancy; oxytocin; electrical stimulation; prostaglandins; sheep

\section{Introduction}

Two patterns of uterine contractile activity occur during pregnancy in sheep and in other species, including humans (Csapo \& Sauvage, 1968), monkeys (Germain et al., 1982), guinea-pigs (Porter, 1971), rats (Fuchs, 1969) and rabbits (Demianczuk et al., 1984). Throughout most of pregnancy in sheep, contractions (termed contractures by Nathanielsz and colleagues (1976)) are of low amplitude $(0 \cdot 27-0.67 \mathrm{kPa})$, long duration $(3-8 \mathrm{~min})$ and low frequency $\left(2-3 \mathrm{~h}^{-1}\right)$ (Hindson \& Ward, 1973; Nathanielsz et al., 1976; Van der Weyden et al., 1981; Harding et al., 1982; Lye et al., 1983; Sigger et al., 1984; Garcia-Villar et al., 1984). Pregnancy contractions are first detected between days 28 and 60 of pregnancy (Van der Weyden et al., 1981; Sigger et al., 1984) and continue until the onset of labour; $>95 \%$ are associated with a burst of myometrial electromyographic activity. In contrast to these contractions during pregnancy, contractions during labour are of high amplitude $\left(>13 \mathrm{kPa}\right.$ ), high frequency $\left(>20 \mathrm{~h}^{-1}\right.$ ) and usually last for $<1 \mathrm{~min}$ (Harding et al., 1982). It is not known whether labour contractions develop out of those occurring during pregnancy or whether these two patterns are generated by two distinct mechanisms. Although most studies have concentrated on the factors that stimulate labour contractions, an understanding of the mechanisms that produce pregnancy contractions may provide critical information on the early switch in activity 
patterns that occurs in premature labour. Despite considerable descriptive data on the occurrence of pregnancy contractions, little is known about their genesis or role in pregnancy. One finding common to all studies of the pregnancy contractions is the constant periodicity (in general $0 \cdot 5-2 \mathrm{~h}$ ) with which they occur in any one animal. Thus, one animal may produce contractions at intervals of $60 \mathrm{~min}$ another at $90 \mathrm{~min}$ and a third at $120 \mathrm{~min}$. Mechanisms proposed to explain this periodicity include (i) an inherent myogenic activity of the entire reproductive tract (Toutain $e t$ al., 1983), (ii) a diffuse stimulation of a nerve net (Harding et al., 1982) and (iii) hormonal stimulation by prostaglandins (El Badry et al., 1984) or systemic oxytocin (Garcia-Villar et al., 1983), although the last group believe this explanation is unlikely since they could not find any correlation between pulsatile release of oxytocin and the occurrence of contractions.

Using a model in which myometrial tissue is dissected from the uterus, attached at its length in vivo to a frame and transplanted to a site in the omental fat, we obtained data to suggest that pregnancy contractions were not due to the pulsatile release of stimulants into the systemic circulation, innervation, or direct interaction with other intrauterine tissues, but were an intrinsic property of the myometrium itself (Lye \& Freitag, 1988). The data of Sigger et al. (1984) support this conclusion.

In this study the mechanism underlying this intrinsic property of the myometrium, allowing it to generate the periodic pattern of contractile activity seen throughout pregnancy, was investigated in relation to possible involvement of (i) prostaglandins in the generation of contractions during pregnancy, (ii) changes in the responsiveness of the muscle to hormonal stimulation, and (iii) evidence for a cyclicity of myometrial excitability that might generate the periodicity.

\section{Materials and Methods}

\section{Surgical preparation}

Nine crossbred sheep of known single insemination dates were used in this study. Surgery was performed at days 110-113 of gestation under general anaesthesia $\left(\mathrm{N}_{2}, \mathrm{O}_{2}\right.$ and halothane). In four of the sheep (Expts 1 and 2), strips $\left(2.5 \times 3.5 \mathrm{~cm}^{2}\right)$ of myometrium alone and endometrium with attached myometrium were removed from the antimesometrial surface of the pregnant uterine horn, attached to stainless steel frames and transplanted to sites within the omental vasculature as described by Lye \& Freitag (1988). In the other five sheep (Expt 3) only myometrial explants were prepared. No significant differences have been found between myometrial and endometrial plus myometrial explants. Two pairs of multifilament, stainless-steel (Cooner Wire, Chatsworth, CA, USA) electrodes were attached at either end of each muscle strip to record electromyographic activity. The electrodes were placed to record voltage differences in the longitudinal aspect of the explants. Reference electrodes were attached to the omental fat.

A catheter was also inserted into the amniotic cavity to enable intrauterine pressure to be monitored. The incisions in the fetal membranes and uterus were closed and a pair of electrodes were sutured in to the antimesometrial surface of the myometrium. Catheters (V11, Bolab) were inserted $20 \mathrm{~cm}$ into the maternal femoral artery and vein such that the catheter tips lay in the abdominal aorta and vena cava, respectively, about $5 \mathrm{~cm}$ proximal to the bifurcation of these vessels.

Post-surgical analgesia and prophylactic antibiotics were given to ewes and fetuses as described by Lye et al. (1983). Catheters were flushed daily with heparinized saline $\left(4 \mathrm{U} \mathrm{ml}^{-1}\right)$, under aseptic conditions.

\section{Protocols}

Experiment 1: contractions and prostaglandins. The role of prostaglandins in the generation of contractions was examined by administration of the prostaglandin synthesis inhibitor, sodium meciofenamate, dissolved in sterile saline $\left(12 \mathrm{mg} \mathrm{mi}^{-1}\right)$ and infused at $600 \mu \mathrm{g} \mathrm{min}^{-1}$ via the femoral vein for $36 \mathrm{~h}$. Spontaneous activity of the explant was assessed during the control saline infusion period $\left(36 \mathrm{~h}\right.$ at $3 \mathrm{ml} \mathrm{h}^{-1}$, via the femoral vein) and during the $36 \mathrm{~h}$ of the sodium meclofenamate infusion. Oxytocin responsiveness was assessed by administering 50,100 or $200 \mathrm{mU}$ of the hormone as a bolus injection via the femoral vein catheter on the day before administration of sodium meclofenamate or after $24 \mathrm{~h}$ of infusion. Each injection was administered about $5 \mathrm{~min}$ after a spontaneous burst of electro. myographic activity from the explant. No further oxytocin was administered until spontaneous activity had returned to baseline levels.

Blood samples ( $5 \mathrm{ml}$, from the maternal femoral artery) were collected $24 \mathrm{~h}$ before, immediately before and $24 \mathrm{~h}$ after the start of the sodium meclofenamate infusion to quantify systemic concentrations of 13,14-dihydro-15-ketoprostaglandin $F_{2 a}$ (PGFM) and thereby provide an indication of the effectiveness of inhibition of PG synthesis. 
Experiment 2: myometrial refractoriness to stimulation. Lye \& Freitag (1988) reported that the myometrium exhibits an apparent period of refractoriness to oxytocin, shortly after a spontaneous contraction. In the present study, the spontaneous periodicity of the explant contractions was first determined. Oxytocin $(50 \mathrm{mU})$ was then administered at 1 or $15 \mathrm{~min}$ after a spontaneous contraction. The time elapsed from administration of oxytocin to the onset of electromyographic burst activity in the explant was recorded. Control injections of saline were also administered at similar times after contractions. No further injections were administered for at least one hour after eliciting a response.

Electrical stimulation of the explant was achieved by passing a DC pulse of 20 ms duration down one of the two pairs of electrodes sewn at either end of the explant myometrium. The sensitivity of the explant was tested $>15 \mathrm{~min}$ after a spontaneous EMG burst and the response was monitored with the remaining pair of electrodes. If no response was elicited the voltage was increased and the myometrium stimulated after the next spontaneous EMG burst. The polarity of the stimulus was alternated between tests. The threshold voltage was defined as the lowest voltage eliciting a response. The threshold voltage was then used to stimulate the explant $1-2 \mathrm{~min}$ after a spontaneous contraction.

Experiment 3: cyclicity of electrical stimulation. Multiple stimulations of the explant myometrium were conducted to define more closely the electrical response characteristics of myometrium during this refractory period. The electrical stimulation threshold was determined by increasing the voltage of the $20 \mathrm{~ms} \mathrm{DC}$ pulses until an electrical response was elicited (usually a single compound action potential). This voltage was subsequently increased or decreased slightly at intervals of $30 \mathrm{~s}$ to determine the threshold for electrical stimulation. During periods of contractions single bursts of electromyographic activity are separated by intervals of $1-3 \mathrm{~s}$, particularly towards the beginning or end of the contraction. The stimulations were conducted during these intervals. Since the response to electrical stimulation is within I $\mathrm{s}$ it was possible to determine the threshold during all but the most intense period of firing. The polarity of the stimulating electrodes was reversed after each stimulus. In addition, the voltage was not increased above $25 \mathrm{~V}$ to minimize the possibility of tissue damage. Each stimulus was analysed in terms of voltage administered and the presence or absence of a response. Stimulations were conducted at intervals of $1-4$ days until the onset of labour. The higher frequency of electromyographic bursts during labour precluded measurement of threshold cyclicity at that time.

\section{Recording of electromyographic activity in vivo}

Electromyographic activity from explants reaches a stable pattern within 10 days of surgery (Lye \& Freitag, 1988). Consequently, EMG activity from the explants and uterus, and intrauterine pressure (from the amniotic fluid catheter) was monitored continuously from day 124 to the end of the experimental period. The electromyographic signal from the uterus and explants was processed through a Grass wide-band AC preamplifier, Model 7P511 J using a half-amplitude low-frequency filter of $0 \cdot 3-1 \cdot 0 \mathrm{~Hz}$ and a half-amplitude high-frequency filter of $10 \mathrm{kHz}$. The signals were recorded using a Grass 78 polygraph. An EMG burst from the explant was defined as a continuous firing of EMG electrodes of amplitude $>50 \mu \mathrm{V}$ and a duration of $>30 \mathrm{~s}$ separated by at least $15 \mathrm{~s}$ from a previous burst. Spontaneous electrical activity was analysed as the frequency and duration of electromyographic bursts. Oxytocin responses were quantitated as the multiple of these two measurements. Electrical stimulation responses were defined as a single or multiple firing of the EMG electrodes occurring within $1 \mathrm{~s}$ of the stimulus (measured from the stimulus artefact).

\section{Prostaglandin measurements}

The concentrations of PGFM in plasma were determined by specific radioimmunoassay that has been fully validated for sheep plasma (Olson et al., 1984). The intra- and interassay coefficients of variation were $<5$ and $<11 \%$, respectively.

\section{Statistical analysis}

All data are expressed as means \pm SEM. Differences between treatments were examined using either a paired $t$ test or a one-way analysis of variance as appropriate. After ANOVA, differences between means were tested using Duncan's multiple range test (set at a level of $P=0.05$ ) after confirming homogeneity of variance.

\section{Results}

\section{Experiment 1}

Administration of sodium meclofenamate significantly reduced the circulating concentrations of PGFM but had no effect on either the frequency or duration of burst of electromyographic activity (contractures) in the explants (Fig. 1). Bolus administration of oxytocin produced a significant dose-related increase in electromyographic response from the explants (Fig. 2); but there 
was no difference between the responses to oxytocin before or during the infusion of sodium meclofenamate. There was also no difference in the response of explants containing endometrium and myometrium from those with myometrium alone.
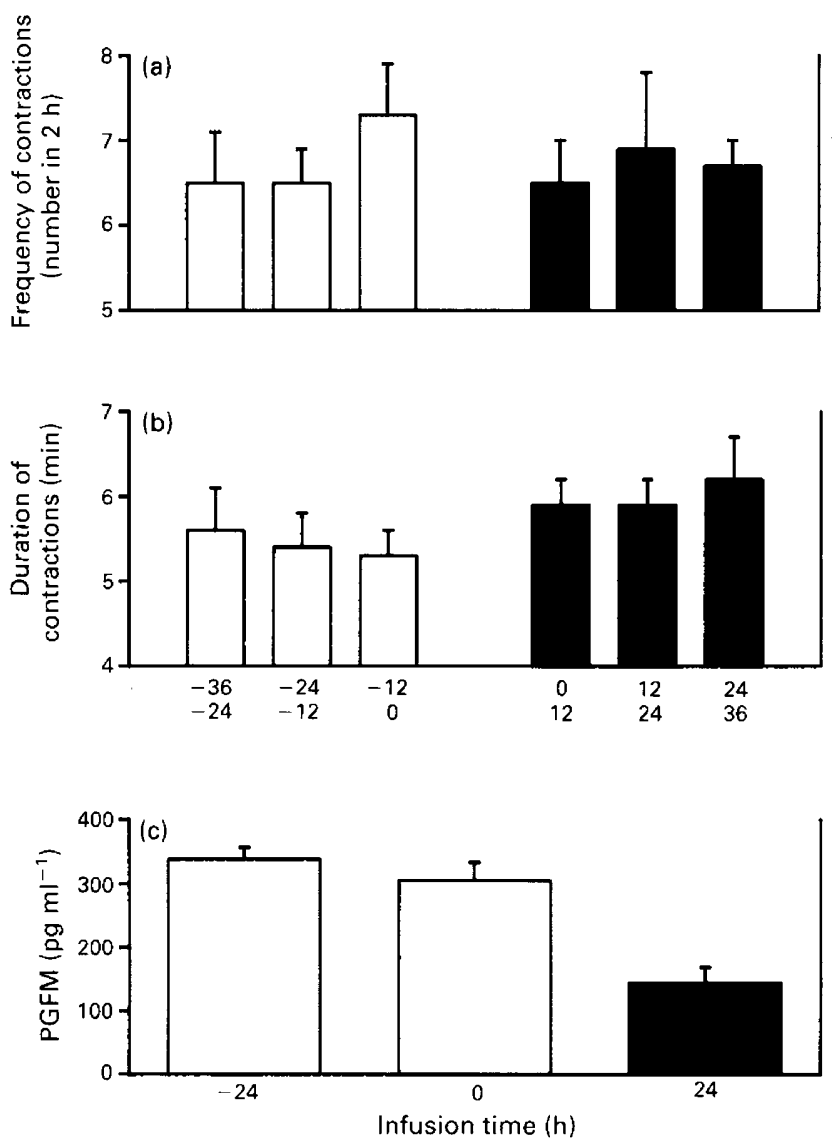

Fig. 1. (a) Frequency and (b) duration of contractions in myometrial explants, and (c) concentration of 13,14-dihydro-15-keto prostaglandin $F_{2 a}$ (PGFM) before ( $\square$ ) and during (-) administration of the prostaglandin synthesis inhibitor sodium meclofenamate. Data are means \pm SEM for four sheep at each time point.

\section{Experiment 2}

A significant delay was observed in the time from bolus injection to the electromyographic response $(8 \cdot 6 \pm 3 \cdot 8$ versus $1 \cdot 3 \pm 0 \cdot 3 \mathrm{~min})$ when oxytocin was administered 1 min versus $15 \mathrm{~min}$ after a spontaneous contraction (Fig. 3). Control injections of saline produced response times significantly different from the paired oxytocin values and were consistent with values for regular intervals between spontaneous contractions (17-20 $\mathrm{min})$ in the explants. Electrical stimulation of the explants also provided evidence that suggested a period of refractoriness. The explant failed to respond to a $3 \mathrm{~V}$ stimulus administered a few minutes after a spontaneous burst of electromyographic activity (Fig. 4), but did respond when the same voltage was administered much later in the inter-contraction interval (i.e. close to the expected time of the next spontaneous contraction). 


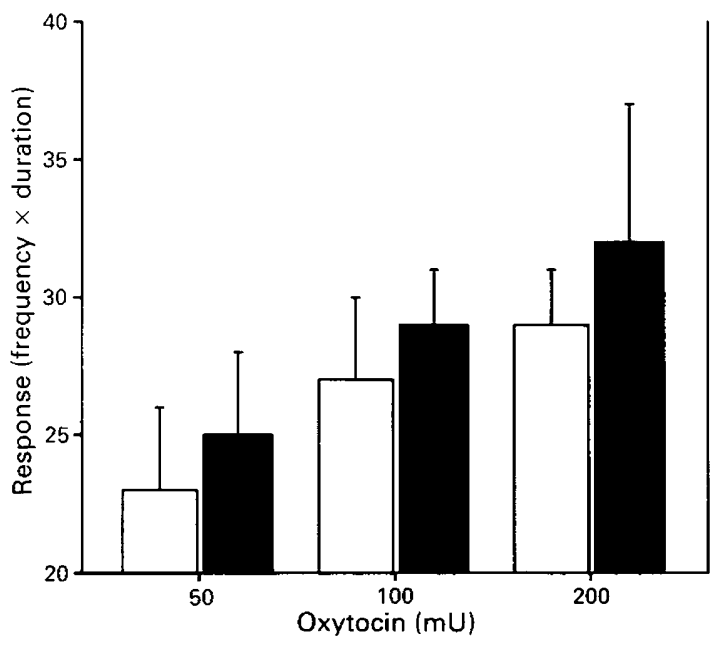

Fig. 2. Dose-response (50-200 mU) stimulation of ovine explant myometrium by exogenously administered oxytocin, before ( $\square$ ) and during ( $\square$ ) the infusion of sodium meclofenamate. Data are means \pm SEM for four sheep at each dose.

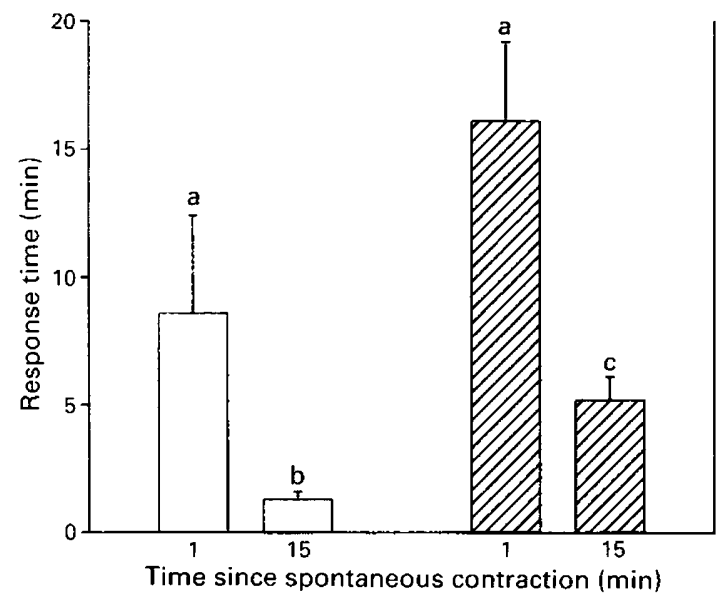

Fig. 3. Time from administration of a bolus of oxytocin ( $\square$ ) or $\mathrm{NaCl}$ ( V//d ) via the femoral vein catheter to eliciting a contractile response in ovine explant myometrium. There was a significant delay in the response time when oxytocin was administered at 1 min versus $15 \mathrm{~min}$ after a spontaneous contraction. Data are means \pm SEM for four sheep at each time point. Bars with different letters above are significantly different from each other $(P<0.05)$.

\section{Experiment 3}

Continuous measurement of the threshold for electrical stimulation throughout the interval between contractions revealed a striking cyclicity in these values (Fig. 5). The voltage needed to elicit a response in the explant was comparatively high between, but fell abruptly at the time of spontaneous contractions. Data obtained from between 4 and 12 cycles from each of five sheep were analysed. The mean voltages eliciting an electrical response from the myometrial explant of each sheep was used to produce an overall mean voltage. This analysis demonstrated that the mean stimulation threshold during contractions $(11.3 \pm 1.6 \mathrm{~V})$ was significantly $(P<0.01)$ lower than the mean threshold between contractions $(18 \cdot 0 \pm 2 \cdot 2 \mathrm{~V})$. The frequency of stimulations, however, was not sufficient to determine whether the threshold fell before or at the time of the spontaneous 

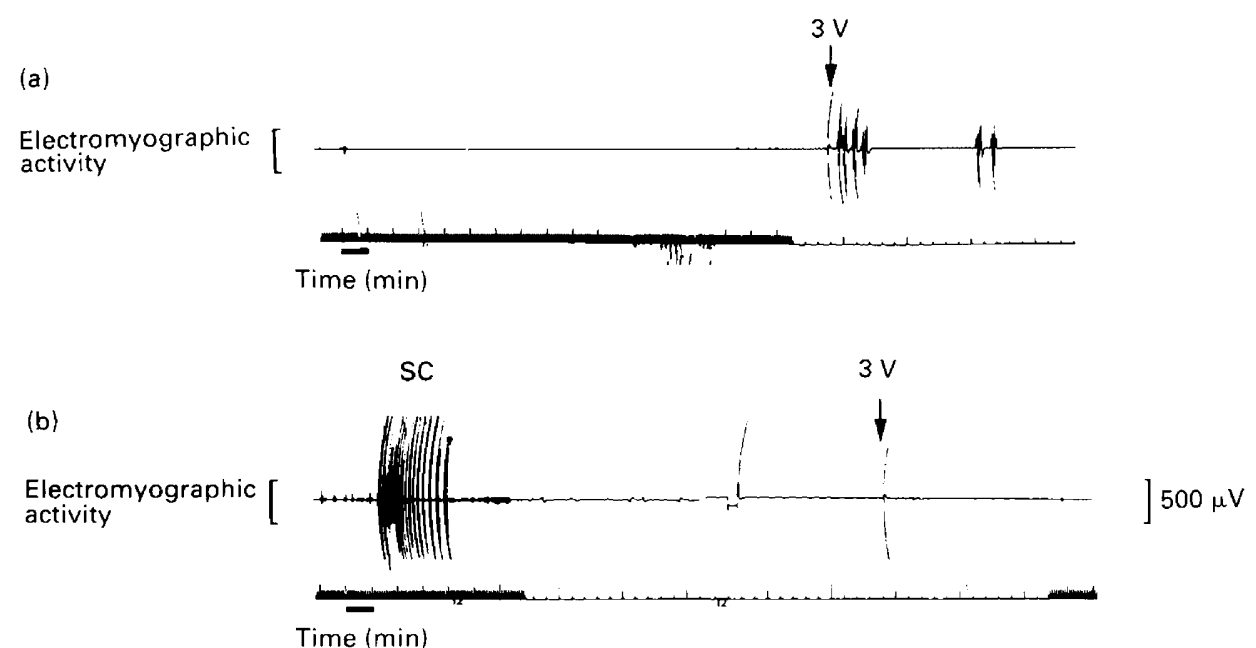

Fig. 4. Continuous recording trace (split into two components) showing the response to stimulus of $3 \mathrm{~V}(20 \mathrm{~ms})$ administered to a myometrial explant, (a) 20 min after a spontaneous contracture (close to the expected time of the next spontaneous contracture), compared with (b) the lack of response when the same stimulus was applied shortly after a spontaneous contracture. The chart speed is run at $1 \mathrm{~cm} \mathrm{~min}$ m $^{-1}$ (the major tick interval of compressed time base and the bar represents $1 \mathrm{~min}$ ) except during the periods around the stimulations when the chart speed is increased (minor tick intervals represent $1 \mathrm{~s}$ ). SC denotes the spontaneous contraction.

contraction. In the period between spontaneous contractions the threshold voltages showed fluctuations; although minor decreases in the stimulation threshold were not associated with the occurrence of spontaneous contractions (Fig. 5). The difference in voltage between and during contractions $(\delta \mathrm{V})$ was somewhat variable and did not change significantly during late pregnancy. However, in the four animals in which measurements were made on the two days before the onset of labour there was a significant fall in $\delta \mathrm{V}$ (day $-2: \delta \mathrm{V}=6.6 \pm 1 \cdot 2 \mathrm{~V}$ versus day $-1: \delta \mathrm{V}=0.68 \pm 1 \cdot 1 \mathrm{~V}$ ).

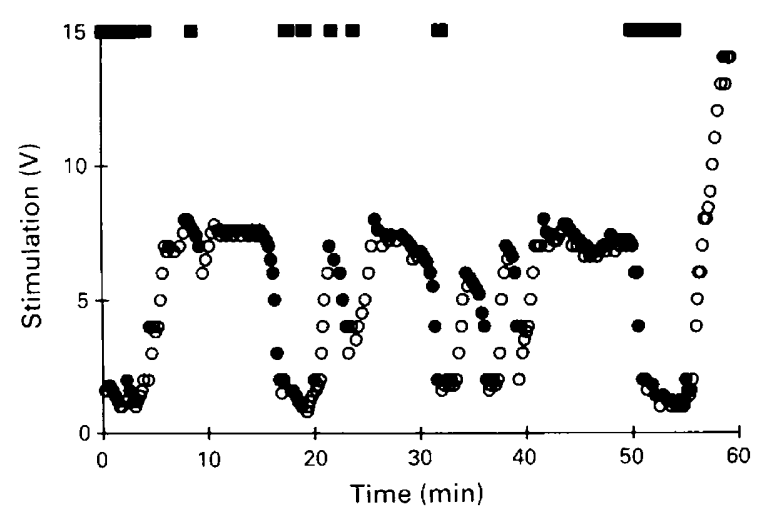

Fig. 5. Changes in stimulation threshold during a series of intervals between contractions; $(\bullet)$ stimuli that elicited responses, $(O)$ stimuli that failed to elicit a response, $(\mathbb{C})$ periods in which spontaneous electrical activity was present in the explant.

\section{Discussion}

Our identification of cyclical changes in the sensitivity of the myometrium to electrical stimulation is the first evidence in vivo to suggest an intrinsic (myogenic) control of myometrial contractions 
during pregnancy. These data are supported by our observations of a period of refractoriness to both hormonal and electrical stimulation of the myometrium shortly after a spontaneous contraction. We suggest that the electrical stimulation threshold may reflect the relative state of excitability of the muscle and suggest that this, in turn, may mirror the resting membrane potential of the myometrial cells. The significantly higher voltage needed to stimulate the myometrium in the inter-contraction period compared with during contractions would be consistent with a generalized hyperpolarization of the myometrium between the contractions. In this study the frequency of electrical stimulation was not sufficient to determine whether the threshold falls before or in association with the onset of the contraction.

Direct electrical stimulation of the uterine smooth muscle in vivo has been used successfully as an experimental tool, but its use is clearly less precise than in vitro techniques using tissues or single cells. Nevertheless, although such in vitro studies would allow direct measurement of electrical potentials, we have previously demonstrated (Lye \& Freitag, 1988) that the characteristic contraction pattern of the myometrium seen in vivo is lost in vitro, thus seriously reducing the rationale for such experiments.

The concept of phasic changes in the excitability of the myometrium is also consistent with observations of spontaneous electromyographic activity. We, and others (Harding et al., 1982), observed that following a spontaneous contraction there is a period of relative quiescence after which single spike activity develops as the next contraction approaches.

The mechanisms that might generate cyclical changes in resting membrane potential are not known, though circumstantial evidence implicates a role for potassium current. Potassium is the most permeant ion and therefore plays a major role in controlling resting membrane potential. Opening $\mathrm{K}^{+}$channels inhibits pace-maker activity in myometrial cells (Hollingsworth et al., 1987), whereas blockade leads to depolarization and can thus increase contractions (Sims et al., 1985). In addition, oestrogen increases the resting membrane potential and periodicity of contractions (Challis \& Lye, 1986), while it also increases mRNA encoding a protein which induces a slow activating $\mathrm{K}^{+}$current (Boyle et al., 1988). The role of $\mathrm{K}^{+}$channels in the genesis of periodicity of contractions needs to be investigated. The observation that the voltage differential during and between spontaneous contractions is lost shortly before the onset of high-frequency myometrial activity (at labour) also suggests that this measurement may mirror important changes in myometrial function at this time and is consistent with data demonstrating significant changes in membrane potential associated with the onset of labour.

Until the mechanisms that are responsible for generating the activity patterns described here are known, it is not clear whether there is any relationship between the refractory period to oxytocin stimulation and the cyclical changes in electrical response threshold of the myometrium. GarciaVillar et al. (1983) found no evidence that contractions are due to systemic pulse release of oxytocin (which in theory could cause a short-term tachyphylaxis to exogenous oxytocin). However, recent data demonstrating that oxytocin is synthesized in intrauterine tissues during pregnancy (Chibbar et al., 1991; Lefebure et al., 1992) again raises the possibility that this hormone could play a paracrine role in the regulation of myometrial contractile activity.

We found no evidence to support a role for prostaglandins in the generation of the contraction pattern. Similar findings have been reported by Mitchell \& Flint (1978) in sheep in labour and by ourselves in nonpregnant oestrogen-treated sheep (Windmoller et al., 1983) using doses of MFA sufficient to block preterm labour. However, two studies (El Badry et al, 1984; Adrianakis \& Walker, 1989) using 4-aminoantipyrine did report a complete abolition of contractions associated with a significant reduction in plasma prostaglandin levels. It remains to be determined whether these differences relate to an increased potency of 4-aminoantipyrine compared with sodium meclofenamate or additional effects exerted by 4-aminoantipyrine on the myometrium. It is possible that the infusion rate of sodium meclofenamate was inadequate to reduce prostaglandins sufficiently to affect contractions. Although the systemic reductions in PGFM seen in this study do not necessarily reflect the changes in the synthesis of $P G$ in tissue, the ability of similar sodium 
meclofenamate doses to block contractile activity during labour (when the PG synthetic capacity is considerably higher than during pregnancy) suggests this is unlikely. Indeed if this explanation is correct, it would suggest that during pregnancy the myometrium is highly responsive to PGs (in that it can generate contractions under conditions of very low PG synthesis), whereas at labour very high levels of PGs must be maintained to maintain labour contractions. This would be in contrast to the considerable literature on the responsiveness of the myometrium during pregnancy and under progesterone domination (Challis \& Lye, 1986).

No significant difference was found between oxytocin stimulated activity before or during infusion of sodium meclofenamate. These data confirm our previous findings in nonpregnant sheep (Windmoller et al., 1983), that the action of oxytocin on the pregnant myometrium is not solely mediated by prostaglandins. Furthermore in those explants with endometrial tissue attached there was also no difference in the response to oxytocin either in the presence or absence of sodium meclofenamate suggesting that in this model either the prostaglandin synthetic capacity of the endometrium is compromised or prostaglandin production by the endometrium does not play a major role in mediating the effects of oxytocin on the myometrium. However, we cannot rule out the possibility that sodium meclofenamate does not block the release of PGs during oxytocin stimulation.

These data provide new information suggesting that myometrial contractions throughout pregnancy are due to an intrinsic property of the muscle. Furthermore, the resting membrane potential of the smooth muscle cells may play a role in modulating the regular periodicity of these contractions.

This work was supported by the Medical Research Council of Canada (Program Grant - Birth and Perinatal Development).

\section{References}

Adrianakis, P. \& Walker, D.W. (1989) Effect of inhibiting prostaglandin synthesis in pregnant sheep with 4-aminoantipyrine under normothermic and hyperthermic conditions. American Journal of Obstetrics and Gynecology 161, 241-247.

Boyle, M.B., MacLusky, N.J., Naftolin, F \& Kaczmarek, L.K. (1988) Hormonal regulation of the $\mathrm{K}^{+}$-channel messenger RNA in the rat myometrium during the oestrous cycle and in pregnancy. Nature 330, 373-375.

Challis, J.R.G. \& Lye, S.J. (1986) Parturition. In Oxford Reviews of Reproductive Biology, Vol. 8, pp. 61-129. Ed. J. R. Clarke. Oxford University Press, Oxford.

Chibbar, R., Miller, F.D. \& Mitchell, B.F. (199I) Oxytocin is synthesized in human fetal membranes. 38th Annual Meeting of the Society for Gynecologic Investigation Abstract 7.

Csapo, A.I. \& Sauvage, J. (1968) The evolution of uterine activity during human pregnancy. Acta Obstetrica et Gynecologica Scandinavica 47, 181-212.

Demianczuk, N., Towell, M.E. \& Garfield, R.E. (1984) Myometrial electrophysiological activity and gap junctions in the pregnant rabbit. American Journal of Obstetrics and Gynecology 149, 485-491.

El Badry, A., Figuero, J.P., Poore, E.R., Sunderji, S., Mitchell, M.D. \& Nathanielsz, P.W. (1984) Effects of fetal intravascular 4-aminoantipyrine infusion on myometrial activity (contractures) at 125 to 143 days' gestation in the pregnant sheep. American Journal of Obstetrics and Gynecology 150, 474-479.

Fuchs, A-R. (1969) Uterine activity in late pregnancy and during parturition in the rat. Biology of Reproduction 1, 344-353.

Garcia-Villar, R., Toutain, P.L., Schams, D. \& Ruckebusch, Y. (1983) Are regular activity cycles of the genital tract controlled by pulsatile releases of oxytocin. Biology of Reproduction 29, 1183-1188.

Garcia-Villar, R., Toutain, P.L. \& Ruckebusch, Y. (1984) Patterns of electrical activity of the ovine uterus and cervix from mating to parturition. Journal of Reproduction and Fertility 72, 143-152.

Germain, G., Cabrol, D., Visser, A. \& Sultan, C. (1982) Electrical activity of the pregnant uterus of the pregnant cynomologus monkey. American Journal of Obstetrics and Gynecology 142, 513-519.

Harding, R., Poore, E.R., Bailey, A., Thorburn, G.D., Jansen, C.A.M. \& Nathanielsz, P.W. (1982) Electromyographic activity of the non-pregnant and pregnant sheep uterus. American Journal of Obstetrics and Gynecology 142, 448-457.

Hindson, J.C. \& Ward, W.R. (1973) Myometrial studies in pregnant sheep. In The Endocrinology of Pregnancy and Parturition-Experimental Studies on the Sheep, pp. 153-162. Ed. C. G. Pierrepoint, Alpha Omega Alpha, Cardiff.

Hollingsworth, M., Amedee, T., Edwards, D., Mironneau, J., Savineau, J.P., Small, R.C. \& Weston, A.H. (1987) The relaxant action of BRL 34915 in the rat uterus. British Journal of Pharmacology 91, 803-813.

Lefebvre, D.L., Giaid, A., Bennet, H., Lariviere, R. \& Zingg, H. (1992) Oxytocin gene expression in the rat uterus. Science 256, 1553-1555. 
Lye, S.J. \& Freitag, C.L. (1988) An in vivo model to examine the electromyographic activity of isolated myometrial tissue from the pregnant sheep. Journal of Reproduction and Fertility 82, 51-61.

Lye, S.J., Sprague, C.L., Mitchell, B.F. \& Challis, J.R.G. (1983) Activation of ovine fetal adrenal function by pulsatile or continuous administration of adrenocorticotrophin-(1-24). I. Effects on fetal plasma corticosteroids. Endocrinology 113, 770-776.

Mitchell, M.D. \& Flint, A.P.F. (1978) Use of meclofenamic acid to investigate the role of prostaglandin biosynthesis during induced parturition in the sheep. Journal of Endocrinology 76, 101-109.

Nathanielsz, P.W., Ratter, S., Thomas, A.L., Rees, L. \& Jack, P.M.B. (1976) The role and regulation of ACTH in the fetal sheep. In The Fetus and Birth (CIBA Foundation Symposium 47), pp. 73-91. Elsevier Science Publishers, Amsterdam.

Olson, D.M., Lye, S.J., Skinner, K. \& Challis, J.R.G. (1984) Early changes in prostaglandin concentrations in ovine maternal and fetal plasma, amniotic fluid and from dispersed cells of intrauterine tissues before the onset of ACTH-induced pre-term labour. Journal of Reproduction and Fertility 71, 45-55.

Porter, D.G. (1971) Quantitative changes in myometrial activity in the guinea-pig during pregnancy. Journal of Reproduction and Fertility 27, 219-226.

Sigger, J.N., Harding, R. \& Jenkin, G. (1984) Relationship between electrical activity of the uterus and surgically isolated myometrium in the pregnant and non-pregnant ewe. Journal of Reproduction and Fertility 70, 103-114.

Sims, S.M., Singer, J.J. \& Walsh, J. (1985) Cholinergic agonists suppress a potassium current in freshly dissociated smooth muscle cells of the toad. Journal of Physiology 367, 503-529.

Toutain, P.L., Garcia-Villar, R., Hazen, C. \& Ruckebusch, Y. (1983) Electrical and mechanical activity of the ewe during pregnancy and parturition. Journal of Reproduction and Fertility 68, 195-204.

Van der Weyden, G.C., Taverne, M.A.M., Dieleman, S.J. \& Fontline, P. (1981) Myometrial electrical activity throughout the entire course of pregnancy in the ewe. European Journal of Obstetrics, Gynaecology and Reproductive Biology 11, 347-354.

Windmoller, R., Lye, S.J. \& Challis, J.R.G. (1983) Estradiol modulation of ovine uterine activity. Canadian Journal of Physiology and Pharmacology 61, 722-728.

Received 16 September 1991 